\title{
Efficacy and Acceptability of a New Water-Soluble Nail Strengthener Containing Pistacia lentiscus and Hyaluronic Acid to Improve the Appearance of Brittle Nails versus Untreated Nails: In vitro and Clinical Evidence
}

\author{
Corinne Granger $^{a}$ Michela Starace ${ }^{b}$ Aurora Alessandrini ${ }^{b}$ Sonia Aladren ${ }^{a}$ \\ Francesca Bruni $^{b}$ Mridvika Narda ${ }^{a}$ Paola Peruginic Bianca Maria Piraccini ${ }^{b}$ \\ annovation and Development ISDIN, Barcelona, Spain; ${ }^{b}$ Division of Dermatology, Department of Experimental, \\ Diagnostic and Specialty Medicine, University of Bologna, Bologna, Italy; ' ${ }^{\mathrm{C}}$ Department of Drug Sciences, University \\ of Pavia, Pavia, Italy
}

\section{Keywords}

Brittle nails · Weak nails · Therapy · Nail fragility · Keratin

\begin{abstract}
Introduction: Brittle nail syndrome is characterized by dry, weak, easily breakable nails that show inelasticity, splitting and overall nail plate fragility. Objective: This paper describes in vitro and clinical studies using bovine hooves designed to assess the efficacy, compatibility and acceptability of a water-soluble nail strengthener (WSNS), containing Pistacia lentiscus and hyaluronic acid, for the treatment of patients affected by brittle nails. Methods: In the in vitro study, the WSNS was tested versus placebo and a marketed product on bovine hoof membranes, applied once daily for 14 days for evaluation of firmness, viscoelasticity and thickness before and after applications. In a clinical study, 23 female subjects affected by brittle nails were included, and the WSNS was applied once daily for 3 months. Signs and symptoms were rated by the investigators and by the participants during treatment at 28 and 84 days with different methods and dermoscopic images. Subjective efficacy was evaluated
\end{abstract}

(c) 2020 S. Karger AG, Basel

karger@karger.com

www.karger.com/sad by specific questionnaires. Results: In the in vitro study, the daily application of the WSNS and marketed product led to a significant increase in firmness index. In the clinical study, the nails on the treated hand presented an improvement in nail appearance and weakness, after 28 and 84 days of WSNS application, respectively. The nails not treated showed no improvement. The WSNS was well tolerated. Conclusions: The WSNS containing Pistacia lentiscus and hyaluronic acid was shown to significantly increase nail firmness in the in vitro study. In the clinical study, WSNS significantly improved brittle nail appearance. The hydrosolubility and easy-to-apply format of WSNS were reported to be factors of a good compliance. We consider this product as an important tool to improve and reinforce the aspect of brittle nails.

(c) 2020 S. Karger AG, Basel

\section{Introduction}

Although the well-being of the nail unit is often overlooked, nail disorders comprise approximately $10 \%$ of all dermatologic conditions. In the USA alone, as many as 
60 million people may be affected by this disorder, with women affected twice as often as men [1]. The incidence in the European and North American population is estimated to be around $20 \%[2,3]$. As such brittle nails are one of the most prevalent nail disorders, characterized by increased fragility of the nail plate due to damage to the nail matrix or changes to nail plate cohesion, caused by internal and/or external factors. This can significantly impair daily activities, occupational abilities and have a major social impact [4].

The diagnostic criteria of brittle nails are clinically characterized by two pathologic abnormalities: excessive onychorrhexis (longitudinal ridging) that results in distal splitting and onychoschizia (horizontal layering) [5]. More recently, brittle nails due to keratin degranulation with superficial friability of the nail plate have been described [6]. Although brittle nails are often perceived as a minor concern with limited aesthetic relevance, when the dystrophy is severe, nail function may become impaired. In addition, it can be a sign of trauma or systemic issues. Determining the root cause of it is useful not only to prevent future damage, but also to rule out serious underlying disorders. The most important causes of brittle nails are believed to be traumatic or physical; however, it could be linked to the deficient production of intercellular cement substance [7]. Idiopathic nail brittleness occurs almost exclusively in fingernails on the first three fingers of the dominant hand [8], with typical clinical presentation described as longitudinal ridging, transverse splitting, horizontal lamellar separation and fissuring of the distal nail plate due to a defect in the intercellular cement responsible for the adherence of the nail plate corneocytes [7,9-11]. The intercellular cement substance is mostly made of phospholipids, mucopolysaccharides and acid phosphatases, and is found in high concentrations around cell junctions, known as the zonula occludens and gap junctions, and makes nail cells firmly adhere to each other [12]. The normal nail plate contains up to $10 \%$ sulfur by weight, $10-18 \%$ water and less than 5\% lipids [13]. Brittle nails are characterized by a loss of organization and/or changes in chemical composition such as dehydration or changes in lipid concentrations. A multitude of regimens exist for the treatment of brittle nails, including buffing and moisturizing, application of essential fatty acids and ingestion of different vitamins, such as biotin, and nutritional supplements [1].

The nail grows slowly, so, when prescribing treatment regimens, the continued compliance of the subjects over a long period of time required to see a clinical improve-

A New Water-Based Nail-Strengthening

Solution for Weak Nails ment in nail fragility is often a major obstacle for successful treatment of the condition. Many studies on topical products about nail fragility are previously tested in vitro on bovine hooves to understand their capacity to induce improvement on the nail plate.

The aims of the studies were to evaluate the efficacy, compatibility and acceptability of a new water-soluble nail strengthener formulation (WSNS) containing Pistacia lentiscus (mastic oil) and hyaluronic acid in an in vitro study where the products were applied once daily for 14 days and a clinical study where the product was applied once daily for 3 months. The effectiveness and the safety of WSNS applied for 3-6 months versus untreated control have been demonstrated in 52 subjects at 3 months and 30 subjects at 6 months on nail brittleness and tendency to breakage [14].

\section{Materials and Methods}

\section{Products Tested}

The product tested is a WSNS specially formulated with a combination of active ingredients (silanediol salicylate and Pistacia lentiscus gum) that help to improve the level of silicon and keratin in the nails. It contains cationic hyaluronic acid that deeply moisturizes the nails and cuticles. This combination of ingredients (patent pending) helps the natural process of nail growth. It was applied on the nails and cuticles around the nails and was randomly assigned for the treated hand; the other hand was not treated (the nails evaluated for each hand having the same level of fragility severity before treatment). The vehicle used in the in vitro study was the WSNS minus silanediol salicylate, $P$. lentiscus gum and cationic hyaluronic acid. A marketed product also tested in the in vitro study was a hydrosoluble nail lacquer with Equisetum extract available for brittle nails.

\section{In vitro Study}

Sixty bovine hooves of about $400 \mu \mathrm{m}$ thickness were obtained and mounted on suitable supports made of polytetrafluoroethylene so that they remained fixed throughout the experiment. The membranes were maintained in a climatic chamber at $30^{\circ} \mathrm{C}$ and $40 \%$ relative humidity for all experiments. Membranes were characterized for morphology, thickness, firmness and viscoelastic behavior in order to start with homogeneous membranes. The membranes were divided into 3 groups: (1) vehicle, (2) WSNS and (3) marketed nail lacquer.

Product Application

For each group the products were applied every $24 \mathrm{~h}$ with the same type of brush; before each application membranes were cleaned with soap, rinsed with water following a specific protocol and left to dry in a conditioned room.

Instrumental Evaluation

At the beginning, and after 14 applications, the following instrumental evaluations were carried out: 
Table 1. Changes in viscoelasticity index (VI), firmness index (FI) and thickness (TH) in bovine hoof-derived membranes after 14 days of treatment with placebo, water-soluble nail strengthener (WSNS) and Equisetum serum nail lacquer (ESNL)

\begin{tabular}{|c|c|c|c|c|c|c|c|c|c|}
\hline & \multicolumn{2}{|l|}{ VI } & \multirow{2}{*}{$\begin{array}{l}\Delta \mathrm{VI} \\
(\mathrm{Tf}-\mathrm{T} 0), \%\end{array}$} & \multicolumn{2}{|l|}{ FI } & \multirow{2}{*}{$\begin{array}{l}\Delta \mathrm{FI} \\
(\mathrm{Tf}-\mathrm{T} 0), \%\end{array}$} & \multicolumn{2}{|l|}{$\mathrm{TH}, \mu \mathrm{m}$} & \multirow{2}{*}{$\begin{array}{l}\Delta \mathrm{TH} \\
(\mathrm{Tf}-\mathrm{T} 0), \\
\%\end{array}$} \\
\hline & T0 & $\mathrm{Tf}$ & & T0 & $\mathrm{Tf}$ & & T0 & Tf & \\
\hline Placebo & $57.62 \pm 13.71$ & $62.13 \pm 12.71$ & 7.81 & $120.43 \pm 37.26$ & $124.09 \pm 48.41$ & 3.05 & $442.52 \pm 38.13$ & $430.62 \pm 33.83$ & -2.69 \\
\hline WSNS & $57.73 \pm 20.45$ & $60.96 \pm 27.12$ & 5.61 & $97.95 \pm 22.14$ & $132.80 \pm 56.65$ & $35.57^{*}$ & $445 \pm 56$ & $433 \pm 51$ & -2.82 \\
\hline ESNL & $53.24 \pm 19.99$ & $59.52 \pm 23.22$ & 11.79 & $96.03 \pm 18$ & $124.78 \pm 49.85$ & $29.94^{*}$ & $459.32 \pm 39.46$ & $438.64 \pm 49.26$ & -4.50 \\
\hline
\end{tabular}

${ }^{*} p<0.05$.

- Viscoelastic Index (VI), obtained from a stress strain curve, by using a Nail StrainStress Meter NM100, set 1 (Courage \& Khazaka, Cologne, Germany)

- Thickness (TH), expressed in micrometers, by using the Nail StrainStress Meter NM100

- Firmness index (FI), obtained from a stress strain curve, by using Nail StrainStress Meter NM100, set 3

All measurements were taken in an air-conditioned room with controlled temperature and humidity on cleaned and dried membranes. The results of each parameter are expressed as means $\pm \mathrm{SD}$ derived from the results of at least 3 measurements on each membrane. Results from all membranes over time are reported, expressed as a mean $( \pm S D)$ of the membrane group. On the data collected, statistical analysis using the $t$ test was carried out. A significance level of $5 \%$ was chosen, so the changes were considered statistically significant for $p<0.05$.

\section{Clinical Study}

This single-center trial in healthy subjects was conducted between April 24, 2019, and July 17, 2019. We included 23 female patients aged between 18 and 65 years with brittle and weak nails with a rough surface and/or tendency to break. For all patients, the diagnosis was established by a trained dermatologist on clinical grounds and from their medical history.

The primary objective was to determine the efficacy through a Hardener nail score of WSNS versus untreated nails having the same degree of impairment documented with dermatoscope images on days 28 (D28) and 84 (D84). The secondary objectives were to evaluate the efficacy improvement through Investigator Global Assessment (IGA) and Patient Assessment Scale (PAS) scores. In addition, a subject self-assessment regarding the efficacy and cosmetic qualities were evaluated through a questionnaire. The compatibility and acceptability of WSNS were evaluated through the dermatologist evaluation and reporting from subjects.

\section{Clinical Evaluations}

The evaluations of treatment efficacy, compatibility and acceptability were assessed at baseline, and after 28 days and 84 days of treatment. The study evaluated the fragility of target nails (treated or untreated) according to the investigator's assessment using a 4-point scale of the hardener nail score (evaluation of nail fragility): 0 = clear of any sign of splitting, ridging or grooves, normal thickness of the nail plate; $1=$ mild tendency to breakage, very few distal horizontal splits and longitudinal grooves; 2 = moderate tendency to breakage with horizontal splits of the nail, few deep ridges and longitudinal grooves, distal longitudinal fissures of the nail plate free edge, mild thinning of the nail; $3=$ severe lamellar or transversal splitting, more than $70 \%$ of the nail showing deep ridging and grooves, extended longitudinal fissures of the nail plate margin, dramatic reduction of the nail plate thickness. In addition, IGA and PAS of treatment efficacy were performed with a specific questionnaire with a scale from 0 to 3 , where 0 indicated no improvement, 1 some improvement, 2 clear improvement and 3 very good improvement evaluated by investigator and subjects, respectively. During each visit, the patient's nails were evaluated with photography and onychoscopic images obtained with standard illumination (one photograph per subject of the most representative nail per hand, severity should be similar between the hands), and the subject reported an assessment of the hardening effect and product cosmetic qualities of the WSNS with a 4-point qualitative scale (ranging from strongly disagree to strongly agree).

Finally, the acceptability was checked every day, by the subjects at home and controlled by the dermatologist, and after questioning of the subjects, while the compatibility of the product was checked after visual examination of the experimental area, by the dermatologist.

\section{Statistical Analysis}

The values are expressed as the mean and standard deviation. A Wilcoxon test for paired samples was performed to evaluate changes between the data obtained at baseline and at each time point. Differences were considered significant at $p<0.05$. Statistical analysis was performed using SPSS software (version 20.0; SPSS Inc., Chicago, IL, USA).

\section{Results}

\section{In vitro Study}

The mechanical data obtained from membranes in groups 1, 2 and 3 at time T0 and Tf (after 14 days treatment) are presented in Table 1 . There were no statistical differences in FI, VI and TH between the three groups at baseline confirming the homogeneity of the tested membranes. After 14 days of treatment both the product WSNS and marketed product with Equisetum extract sig- 


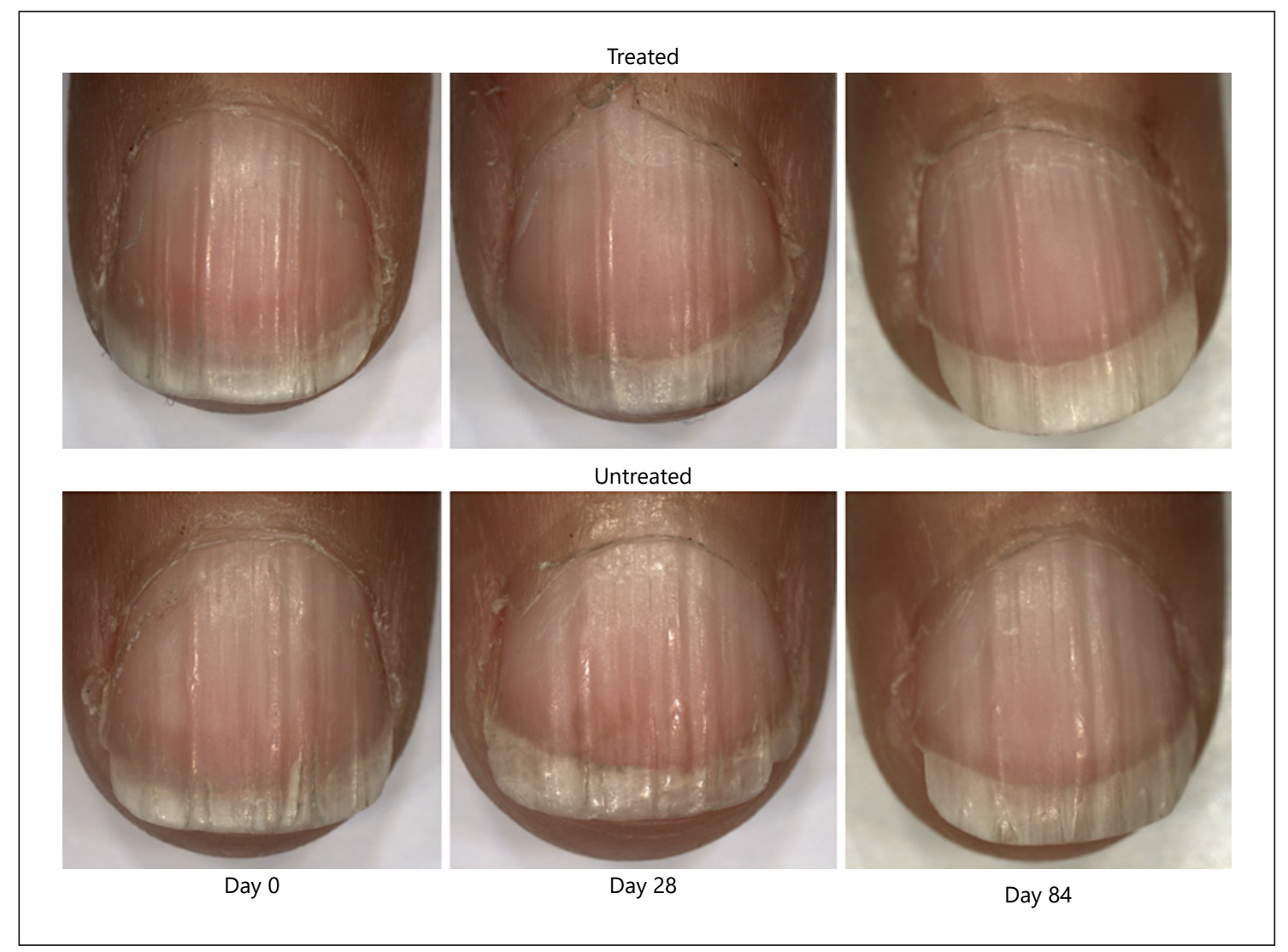

Fig. 1. Dermoscopy images of patient 12, treated nail hand and untreated nail hand, at baseline, D28 and D84.

nificantly increased the FI. This parameter increased from $97.95 \pm 22.14$ to $132.80 \pm 56.65$, with a $35.57 \%$ increase $(p=0.0389)$ in the case of WSNS. The vehicle group had a nonsignificant increase of $3.05 \%$ and the marketed product nail lacquer with Equisetum extract also had a statistically significant increase in FI of 29.94\% $(p=0.0487)$. Changes in the other two parameters VI and TH were nonremarkable (Table 1 ).

\section{Clinical Study}

Twenty-three female volunteers were enrolled for 3 months of treatment, aged $38.4 \pm 15.10$ years. Three patients dropped out for personal reasons, 2 patients before the D28 visit and 1 patient before the last visit on D84. Therefore, 21 subjects were evaluated during the $\mathrm{D} 28$ visit and 20 subjects on D84. The product was applied once daily on the treated nails and their cuticles.

\section{Clinical Evaluation of Nail Hardness}

The hardener nail score of treated nails showed statistically significant decreases at D28 and D84 versus D0
( $p<0.05)$, respectively, while the hardener nail score of untreated nails presented similar values through the entire study. The product application induced 15.9 and $26.7 \%$ improvement in nail hardness of treated hands after 28 and 84 days versus $\mathrm{D} 0$, respectively. In addition, the hardener nail score of treated nails was compared to the untreated nails at the same time point after treatment, the results showed a statistically significant improvement for the hardener nail score for the treated nails $(p<0.011)$ at D28 and D84 (Fig. 1).

\section{IGA Evaluation}

As a result of the application, there was a considerable improvement as shown by the IGA score. The average IGA score in the treated hand presented a statistically significant increase of 0.8 at D28 and 1.3 at D84 versus only 0.3 in the control hand. Investigators reported 76.2 and $95 \%$ of subjects with improvement of nail hardness on the treated hand versus 0 and $25 \%$ of subjects with improvement on the untreated hand at D28 and D84, respectively. From this improvement, the investigator reported clear 
improvement in the treatment hand, with 4.8 and $30 \%$ of subjects at D28 and D84, respectively.

The improvement evolution evaluated by the investigator of treated nails is statistically significant $(p<0.001)$ versus untreated nails for both visits.

Onychoscopy images objectify the results obtained on the improvement of the nail fragility (Fig. 1).

\section{Patient Global Assessment Evaluation}

The patients evaluated the improvement of the nail after application of the product through PAS. In the study, on the treated hand after 28 days, $9.5 \%$ of subjects noticed no improvement, $47.6 \%$ some improvement, $23.8 \%$ a clear improvement and $19.0 \%$ a very good improvement, while after 28 days, on the untreated hand $85.7 \%$ of subjects noticed no improvement and $14.3 \%$ some improvement.

After 84 days, as a result of the product application on the treated nails, $20.0 \%$ of subjects noticed some improvement, $60.0 \%$ a clear improvement and $20.0 \%$ a very good improvement. After 84 days, on the untreated nails $100.0 \%$ of subjects noticed no improvement.

The improvement evolution of treated nails evaluated by the patient is statistically significant $(p<0.001)$ versus untreated nails for both visits.

\section{Self-Evaluation Questionnaire}

In general, after completing the questionnaire about cosmetic qualities after 28 and 84 days, all patients defined the product as easy-to-apply and to use with applicator packaging, with a pleasant fragrance and texture and without leaving residues or sensation on the nail. After completing the questionnaire about efficacy at 28 and 84 days, almost all of the patients had excellent compliance and defined the nails as harder, hydrated, cared, with a smooth surface that reflected a better aspect, and effective with reduction of breaks from the beginning and an improvement of cuticles. In addition, $90 \%$ of the subjects would buy the product and 95\% would recommend the product to other individuals on D28 and D84. At the same time, $95.0 \%$ of the subjects had a positive opinion of the product, and these results reflect the good compliance reported in this study.

\section{Tolerance and Safety}

Under the experimental conditions adopted, no skin reaction was noted after the application of the product. No subject experienced any discomfort during the study. Therefore, the products presented a very good skin and nail acceptability and compatibility during the study under normal conditions of use.

\section{Discussion}

The efficacy of WSNS in improving the firmness (FI) of nails was demonstrated in bovine hoof membranes, a well-defined model for the human nail which can be used for studying nail permeability and the effect of ingredients on nails [21-23]; after 14 days of treatment, it showed a $38.6 \%$ increase in firmness compared with the vehicle. In the clinical study the treated nails showed statistically significant improvement in hardener nail scores at D28 and D84 versus D0, respectively. In addition, the product application led to a significant improvement in the IGA score (0.8 at D28 vs. 1.3 at D84). These changes were accompanied by a clear improvement of nail conditions reducing nail fragility as evidenced by onychoscopy images. These improvements were assessed positively by the patients through PAS after 28 and 84 days of treatment when $100 \%$ of patients reported some, clear or very good improvement in the quality of nails. These results are in agreement with previously published efficacy data obtained after 3 and 6 months of treatment in an open clinical study evaluating WSNS in brittle nails [14].

Brittle nails may be idiopathic or secondary to factors that alter nail plate production and/or damage on the already keratinized nail plate [13]. Regardless of the causative factors involved, the splitting, flaking, nail plate fragility, crumbling and low elasticity of these nails tends to interfere in daily life activities and thus impact the quality of life of these patients. Treatment of brittle nails is not easy and multiple approaches are essential. Therapeutic approaches should include eliminating the eliciting factors that usually include limiting contact with water, chemicals and detergents as much as possible. Protective measures include cosmetic treatments that induce moisturizing and hydration such as nail hardeners, nail lacquers and nail-strengthening agents which form an important part of the overall therapeutic approach.

The nail plate is a unique structure that provides hardness, flexibility and elasticity to the nail which is essential for its function [10]. The nail plate is composed mainly of proteins such as hard and soft keratins, mucopolysaccharides and intracellular cement rich in sulfur. The hard and soft keratins are an essential part of the nail as they ensure hardness and elasticity of the nail plate $[15,16]$. WSNS composition includes mastic oil, an aromatic resin produced by the plant $P$. lentiscus that has previously been shown to stimulate the synthesis of hard keratins and keratin-associated proteins, increasing nail thickness and strength [14]. 
The overall sulfur content of the nail is approximately $10 \%$ by weight and contributes largely to nail hardness by acting as the glue that holds the keratin fibers together, thereby creating the nail plate's tensile strength. Exogenous silicon supports nail growth and improves the nail appearance. Silicon is also a normal component of the skin, and its addition to the periungual tissues reinforces their normal growth.

The hydration state of the nail is another important factor for nail hardness. The nail plate's water content can vary greatly, with the normal content being around $18 \%$ [17]. Nails tend to become brittle when the water content is less than $16 \%$ and soft when this percentage is greater than 25\% [18]. In brittle nails, marked dilatation of wider intercellular spaces was frequently observed and electrodense layers were either not seen or were disrupted. Dehydration may result in a defect of intercellular dilatation of corneocytes that results in brittle nails [19]. Environmental factors, such as excessive contact with water or chemicals, age and gender are important factors that affect the level of nail dehydration [20]. The product WSNS contains hyaluronic acid to boost the hydration of the nail plate reducing the dilatation of the intercellular spaces, and this capacity could explain its efficacy after only 3 months of application.

One of the most important considerations for treatments of brittle nails is that they require time and patience on the part of the patients as they need to be consistent with the treatment for a rather long period (typically $>3$ months) before evidencing an improvement in nail quality and condition. In case of WSNS, the easy-to-apply format was valued positively by all subjects that helped compliance with the daily application of the treatment. In a previous study, strengthening and hardening properties of the hydroxypropyl-chitosan nail lacquer, also containing Equisetum arvense, and methylsulfonylmethane were shown to improve the structure of the nail plates on psoriatic nails in a 24-week clinical study [24].

One of the limitations of this work was that in a clinical study, the comparison was done versus untreated nails and not versus a placebo product. However, in the in vitro model we have demonstrated that WSNS was statistically superior to vehicle in terms of improving nail hardness. The second limitation of the study is that the investigator evaluating the nails was not blinded. However, we controlled for the potential bias by including dermoscopic photographs that allowed us to have an objective view of the results which are aligned with the previous data obtained.

A New Water-Based Nail-Strengthening Solution for Weak Nails

\section{Conclusion}

In conclusion, treatment with a cosmetic hydrosoluble nail lacquer product containing P. lentiscus and hyaluronic acid significantly improved nail quality and appearance in an in vitro model mimicking human nails and brittle nails in a 12-week clinical study. The nail lacquer was very well tolerated and was positively perceived by patients in terms of the easy-to-apply and pleasant-to-use format and texture. These pieces of evidence suggest that this product will be an important tool in treating brittle nails and the surrounding cuticles, irrespective of their etiology, by rehydrating the nail plate and improving its keratin profile.

\section{Acknowledgments}

We thank the participants of the study.

\section{Statement of Ethics}

All named authors meet the International Committee of Medical Journal Editors (ICMJE) criteria for authorship for this article, take responsibility for the integrity of the work as a whole and have given their approval for this version to be published.

Due to the cosmetic nature of the intended use of the product studied, ethics committee approval was not required. The study was performed in line with the Declaration of Helsinki (1964) and its subsequent amendments. The subjects were informed about the expected benefits of the tested products and the constraints of study participation and signed an informed consent form, including consent for the use of photographs taken prior to the start and during the study.

\section{Disclosure Statement}

Corinne Granger, S. Aladren and M. Narda declare that they are employed by ISDIN. The other authors declare that they have no conflict of interest.

\section{Funding Sources}

Sponsorship for this study and Rapid Service Fee were funded by ISDIN (Barcelona, Spain, VAT: ESA08291924, contact for the invoice: elzbieta.obrok@isdin.com).

\section{Author Contributions}

All the authors contributed to the design, draft and final approval of the work. All authors had full access to all of the data in this study and take complete responsibility for the integrity of the data and accuracy of the data analysis.

Skin Appendage Disord 2020;6:108-114 113 


\section{References}

1 Hochman LG, Scher RK, Meyerson MS. Brittle nails: response to daily biotin supplementation. Cutis. 1993 Apr;51(4):303-5.

2 Lubach D, Cohrs W, Wurzinger R. Incidence of brittle nails. Dermatologica. 1986;172(3): 144-7.

3 Scher RK, Bodian AB. Brittle nails. Semin Dermatol. 1991 Mar;10(1):21-5.

4 van de Kerkhof PC, Pasch MC, Scher RK, Kerscher M, Gieler U, Haneke E, et al. Brittle nail syndrome: a pathogenesis-based approach with a proposed grading system. J Am Acad Dermatol. 2005 Oct;53(4):644-51.

5 Kechijian P. Brittle fingernails. Dermatol Clin. 1985 Jul;3(3):421-9.

6 Iorizzo M, Pazzaglia M, M Piraccini B, Tullo S, Tosti A. Brittle nails. J Cosmet Dermatol. 2004 Jul;3(3):138-44.

7 Colombo VE, Gerber F, Bronhofer M, Floersheim GL. Treatment of brittle fingernails and onychoschizia with biotin: scanning electron microscopy. J Am Acad Dermatol. 1990 Dec; 23(6 Pt 1):1127-32

8 Baran R, Schoon D. Nail fragility syndrome and its treatment. J Cosmet Dermatol. 2004 Jul;3(3):131-7.

9 Wallis MS, Bowen WR, Guin JD. Pathogenesis of onychoschizia (lamellar dystrophy). J Am Acad Dermatol. 1991 Jan;24(1):44-8.

10 Shemer A, Daniel CR 3rd. Common nail disorders. Clin Dermatol. 2013 Sep-Oct;31(5): $578-86$.
11 Chessa MA, Iorizzo M, Richert B, López Estebaranz JL, Rigopoulos D, Tosti A, Gupta AK, Di Chiacchio N, Di Chiacchio NG, Rubin AI, Baran R, Lipner SR, Daniel R, Chiheb S, Grover C, Starace M, Piraccini BM. Brittle nails: etiopathogenesis, clinical signs and treatment recommendations. Dermatol Ther. 2019 (approved)

12 Hashimoto K. Cementsome, a new interpretation of the membrane-coating granule. Arch Dermatol Forsch. 1971;240(4):349-64.

13 Iorizzo M. Tips to treat the 5 most common nail disorders: brittle nails, onycholysis, paronychia, psoriasis, onychomycosis. Dermatol Clin. 2015 Apr;33(2):175-83.

14 Piraccini BM, Granger C, Alessandrini A, Brandi N, Bruni F, Starace M. Clinical and instrumental objective evidence of the efficacy of a new water-soluble nail strengthener formulation containing Pistacia lentiscus and hyaluronic acid applied for up to 6 months to improve the appearance of weak, brittle nails. Dermatol Ther. 2019 (approved)

15 Batory M, Namieciński P, Rotsztejn H. Evaluation of structural damage and $\mathrm{pH}$ of nail plates of hands after applying different methods of decorating. Int J Dermatol. 2019 Mar; 58(3):311-8.

16 Fistarol SK, Itin PH. Nail changes in genodermatoses. Eur J Dermatol. 2002 Mar-Apr; 12(2):119-28

17 Singh G, Haneef NS, Uday A. Nail changes and disorders among the elderly. Indian J Dermatol Venereol Leprol. 2005 Nov-Dec; 71(6):386-92.
18 Cohen PR, Scher RK. Aging. In: Hordinsky MK, Sawaya ME, Scher RK, editors. Atlas of hair and nails. Philadelphia: Churchill-Livingstone; 2000. p. 213-25.

19 Kitamori K, Kobayashi M, Akamatsu H, Hirota-Sakashita A, Kusubata M, Irie S, et al. Weakness in intercellular association of keratinocytes in severely brittle nails. Arch Histol Cytol. 2006 Dec;69(5):323-8.

20 Brosche T, Dressler S, Platt D. Age-associated changes in integral cholesterol and cholesterol sulfate concentrations in human scalp hair and finger nail clippings. Aging (Milano). 2001 Apr;13(2):131-8

$21 \mathrm{Kim} \mathrm{JH}$, Lee $\mathrm{CH}$, Choi HK. A method to measure the amount of drug penetrated across the nail plate. Pharm Res. 2001 Oct;18(10):146871.

22 Monti D, Saccomani L, Chetoni P, Burgalassi $S$, Saettone MF, Mailland F. In vitro transungual permeation of ciclopirox from a hydroxypropyl chitosan-based, water-soluble nail lacquer. Drug Dev Ind Pharm. 2005 Jan; 31(1):11-7.

23 Myoung Y, Choi HK. Permeation of ciclopirox across porcine hoof membrane: effect of pressure sensitive adhesives and vehicles. Eur J Pharm Sci. 2003 Nov;20(3):319-25.

24 Cantoresi F, Caserini M, Bidoli A, Maggio F, Marino R, Carnevale C, et al. Randomized controlled trial of a water-soluble nail lacquer based on hydroxypropyl-chitosan (HPCH), in the management of nail psoriasis. Clin Cosmet Investig Dermatol. 2014 May; 7:185-90. 